\title{
In-patient nutrition counseling and therapy - Exploration of structures for process driven work in a German clinical centre
}

\section{Abstract}

Introduction: An implementation of process driven work in dietetics requires detailed analysis and specific planning. Therefore, gathering information on structures of the institution is an essential step and prerequisite for the development of an implementation concept. Process driven work in nutrition counseling and therapy, especially the implementation of the new developments into practice becomes more and more important.

The aim of the present qualitative study was to assess and analyse structures of the in-patient setting of nutrition counseling and therapy in a German clinical centre. Subsequently, strengths and challenges for the implementation of process driven work were identified.

Materials and Methods: Based on a structured literature search, guidelines for interviews with dietitians of a clinical centre were developed. The five interviews were recorded, transcribed with $\mathrm{f} 4 \AA$ and analysed by using the qualitative content analysis by Mayring. Finally, strengths and challenges for process driven work were identified out of this qualitative research.

Results: The following strengths and challenges were identified:

Strengths: concrete responsibility for indications, flexible duration of patient-consultations, use of standardized patient information, use of a digital documentation system, continuous intra- and interprofessional exchange, participation in education and training

Challenges: variety of different activities, barriers in the access to the dietitian, often only one-time dietitian-patient-consultation, time dependent problems because of the limited in-patient therapy, deficiency of rooms for a private patient consultation, non-existing data exchange from the in-patient into the outpatient setting, knowledge deficits of dietitians regarding process-driven work

Discussion: For the new developments for process driven work adequate structures of nutrition counseling and therapy in the in-patient setting are very important for patient care. Based on the gathered information on structures and processes, a concept for the implementation of a process model with a target and milestone planning will be helpful. Especially the appropriate access to patients as well as the fact that the dietitian-patient-consultation is mainly one-time have to be considered for the implementation concept. The integration of every process step in the in-patient setting is difficult to realize. Therefore, the prioritization of the considered process steps is necessary. Consequently, an exchange with the outpatient setting is advisable for the implementation of the complete process model.

\section{Conflict of Interest}

There is no conflict of interest. 